\title{
Lexis
}

Journal in English Lexicology

8| 2014

Metaphor Studies in the English Language

\section{La création lexicale d'origine métaphorique dans le discours journalistique sportif}

De la productivité de la structure verbale anglaise Vb+out / off / away et des problèmes de traduction en français

\section{Romain Vanhoudheusden}

\section{(2) OpenEdition}

Journals

\section{Édition électronique}

URL : http://journals.openedition.org/lexis/273

DOI : $10.4000 /$ lexis. 273

ISSN : 1951-6215

Éditeur

Université Jean Moulin - Lyon 3

Référence électronique

Romain Vanhoudheusden, «La création lexicale d'origine métaphorique dans le discours

journalistique sportif », Lexis [En ligne], 8 | 2014, mis en ligne le 09 janvier 2014, consulté le 02 mai

2019. URL : http://journals.openedition.org/lexis/273 ; DOI : 10.4000/lexis.273

Ce document a été généré automatiquement le 2 mai 2019.

\section{(c) $($ i) $(9)$}

Lexis is licensed under a Creative Commons Attribution-NonCommercial-NoDerivatives 4.0

International License. 


\section{La création lexicale d'origine métaphorique dans le discours journalistique sportif}

De la productivité de la structure verbale anglaise $\mathrm{Vb}+$ out / off / away et des problèmes de traduction en français

\section{Romain Vanhoudheusden}

\section{Introduction}

Dans le discours journalistique sportif, les commentateurs se doivent, par une convention qui s'est imposée depuis les débuts de la profession, de suivre un style extrêmement codifié par des usages linguistiques et extralinguistiques précis que nous définissons comme étant stéréotypés [Vanoudheusden 2010]. Ce genre textuel semble trouver ses fondements dans une histoire de la presse sportive particulièrement liée au monde littéraire, tant au niveau des premiers auteurs qu'à la copie du style littéraire. Si l'on peut prétexter qu'il en est de même pour tout genre journalistique, il semble pour autant que le discours journalistique sportif, de par son rôle de commentateurs d'événements passés ou à venir, s'en est beaucoup moins détaché pour différentes raisons, en particulier celle qui voudrait que les journalistes de sport y ont trouvé une certaine reconnaissance aux yeux de leur lectorat et peut-être de leurs pairs journalistes via l'avènement dans les textes de cette filiation.

Dans nos travaux antérieurs, nous avons cherché à dégager les contraintes linguistiques et culturelles qui sont imposées aux journalistes sportifs lors de la production écrite. La description d'éléments répétés et marqués, tant au niveau micro- que macro-structurel nous ont menés à nous interroger sur l'usage de la métaphore en général. Une analyse du spectre métaphorique présent dans le discours journalistique sportif nous a amené à étudié ce genre spécifique via la théorie de la conception cognitive métaphorique de Lakoff et Johnson [1980]. Comme dans les analyses de bon nombre de linguistes s'étant 
penchés sur la question du commentaire sportif, certains traits particulièrement saillants ont retenu notre attention, comme celui parfaitement illustré par les propos de George Orwell en 1946 :

Sport is war, without weapons.

\section{Contexte}

Dans ce type de discours particulièrement structuré, les métaphores sont aussi un moyen de création lexicale particulièrement productif, en particulier en anglais, langue dont la malléabilité a été soulignée de nombreuses fois. Dans ses matrices internes lexicogéniques de l'anglais contemporain, Jean Tournier [2004] relève différents modes de construction néologique dont les deux principales sont l'affixation et la composition.

Puisque le sport est une affaire de compétition, il est aussi, pour le perdant, une histoire d'élimination. Pour cet article notre interrogation portera sur le défi de traduction que représente le passage de l'anglais au français, soit la traduction d'une langue à cadrage satellitaire à une langue à cadrage verbal [Talmy, 1991] de ce type de métaphore construite en anglais par le schéma particulièrement productif qui relève de la métaphore du mouvement traduisant une mise à l'écart, une l'exclusion ou une élimination, $\mathrm{Vb}+$ out/off/away et dont le français n'a pas d'équivalent.

La théorie des métaphores conceptuelles (Conceptual Metaphor Theory) de Lakoff et Johnson [1980] montre à quel point les métaphores sont importantes dans le processus cognitif qui permet de décrire le monde. La Conceptual Metaphor Theory considère que l'étude du langage permet de mieux interpréter notre façon de penser. Les métaphores, par leur caractéristiques spécifiques tant au niveau morphosyntaxique que sémantique, jouent un rôle extrêmement important car leur façon dérivée de décrire le monde permet de conceptualiser tout ce qui nous entoure, et en particulier d'appréhender les concepts abstraits.

We claim that most of our normal conceptual system is metaphorically structured;

that is, most concepts are partially understood in terms of other concepts. [1980 : 56]

L'appréhension des concepts se fait par l'expérience sensorielle et culturelle. Ainsi le concept que l'on tente de comprendre (le concept cible) n'est compris qu'en passant par un autre concept (le concept source).

$6 \quad$ Il ne sera étonnant pour personne de lire que la plupart des études faites sur la langue du sport sont des études sur les emplois métaphoriques. Jusqu'au début des années 2000, il s'agissait en grande partie d'études d'origine germanophone. Pour autant, ces études ne se sont pas cantonnées à travailler sur l'allemand, mais ont développé des analyses contrastives entre les principales langues européennes (anglais, allemand, espagnol, français, italien, auxquels s'ajoute le suèdois). Parmi ces études, le travail lexicographique contrastif du Kicktionary de Thomas Schmidt [2008], une base de données créée à partir d'articles et de traductions du site de l'UEFA, sera à la base du processus de réflexion engagé dans ces quelques lignes.

7 La plupart de ces travaux ont privilégié l'étude du langage des commentateurs sportifs à la télévision ou à la radio en synchrone. Ainsi Michels [2002] et Nordin [2008] ont tous deux travaillé sur des corpus oraux. L'analyse d'un corpus oral de commentateurs lors d'un même match en allemand et en suédois a conduit Henrik Nordin à déterminer différents concepts utilisés métaphoriquement dans le discours du football. Ses 
conclusions l'amènent à une liste non exhaustive de métaphores conceptuelles, classées selon les catégories définies par Lakoff et Johnson et dont les principales traitant directement du sport sont :

- DÉFENDRE CONSISTE À NETTOYER,

- Le fOotball EST LA GUeRRE :

Some examples from my data on the concept FOOTBALL IS WAR:

[...] das ist die Innenverteidigung des FC Bayern [...] ([...] that is the inner defence of

FC Bayern [...])

Jag minns en par räder i EM [...] (I remember a couple of raids in the Euro 2004)

[Nordin 2008: 116]

8 Notre travail effectué dans le cadre de ce numéro sur la métaphore prendra en partie une réflexion menée lors de notre recherche doctorale. Nous exploiterons les articles en ligne de l'UEFA et de la FIFA publiés en anglais et en français. Afin de ne pas se focaliser, de facto , sur le football, nous avons pris le parti d'interroger grâce à des syntaxes avancées les sites Internet du mouvement olympique anglais et français.

9 Le travail de traduction au sein de ces instances se fait principalement dans le sens anglais $\rightarrow$ français excepté dans le cas de sujet traitant d'actualités sportives françaises ou d'origine francophone, écrites par des correspondants francophones.

Pour des raisons de concision, notre choix s'est orienté vers les structures verbales prépositionnelles les plus courantes dans le domaine du commentaire sportif. La liste pourra sembler limitée mais elle permet malgré tout d'esquisser quelques problèmes de traduction et surtout comment ceux-ci sont résolus par les traducteurs travaillant pour les sites Internet cités.

\section{La traduction spécifique}

11 Nous avons ici fait le choix d'interroger une forme spécifique de l'anglais qui n'a pas d'équivalent stricto sensu en français, les verbes suivis d'une particule adverbiale. Puisque le sport est une affaire de compétition, elle est aussi, pour le perdant, une histoire d'élimination; en conséquence, nous avons choisi de restreindre nos recherches sur les particules suivantes : out, off et away. Ce choix a été dicté par leur sémantisme. Nous nous focaliserons sur les emplois spécifiques au domaine étudié, c'est-à-dire que nous avons fait le choix de ne pas recenser et analyser toutes les formes rencontrées mais uniquement celles qui représentent un intérêt syntaxique (différence de traitement dans les deux langues) et stylistique (métaphore en anglais), et ce afin d'interroger la traduction. Ce choix est dicté, comme nous l'avons déjà écrit par des contraintes de place mais également par la volonté de mettre en avant dans ces quelques lignes le travail important d'adaptation qu'implique la traduction dans ce cadre.

Évidemment, les emplois que l'on appellera « standard » peuvent se révéler intéressants, mais ils ne relèvent pas forcément de la métaphore, élément stylistique particulièrement important dans le discours et le commentaire sportif. Ainsi, nous retrouvons en (1') la traduction quasi évidente de (1).

(1) Grønkjær and Jørgensen bow out. - UEFA, le 25 juin 2010.

(1') Grønkjær et Jørgensen arrêtent. - UEFA, le 25 juin 2010.

En anglais, bow out signifie " arrêter de prendre part à une activité » et nécessite un sujet animé actif. Cet exemple permet tout de même de relever une des distinctions 
caractéristiques entre l'anglais et le français comme un cadre théorique et utile en amont de notre description. L'anglais est ce que Talmy [1991] a appelé "a satellite-framed language » alors que le français est « a verb-framed language ». Pour décrire un mouvement l'anglais utilise principalement des verbes avec une particule, la particule portant le sens du mouvement, alors que le mouvement est intégré au verbe en français et dans les langues romanes en général.

\subsection{La traduction dans une langue spécifique}

Les sites Internet des instances européennes et internationales des principales fédérations sportives sont des plate-formes énormément visitées par les amateurs de sport sur le web. Ces sites sont des agrégateurs d'actualités sportives, qui recensent tout ce qui peut se passer dans leur domaine respectif. Ainsi, par exemple, l'Union of European Football Associations (UEFA), organisatrice des compétitions de football, de futsal entre clubs et équipes nationales d'Europe, est une association des fédérations nationales. Le site Internet recense ainsi l'actualité footballistique de chacune des cinquante-quatre fédérations associées mais également celle de tous les championnats professionnels, ainsi que des compétitions que l'UEFA gère.

Il ne nous a pas été possible d'extraire toutes les données, ni même de savoir, même approximativement, de combien de mots ces archives étaient composées à un jour déterminé. Il en est de même pour les archives des sites Internet de la FIFA (Fédération Internationale de Football Association) et du CIO (Comité International Olympique). Du point de vue méthodologique, cela pose un problème évident, car les moteurs de recherche de ces sites ne permettent que rarement des syntaxes avancées performantes comme le ferait n'importe quel concordancier. Face à notre incapacité à parcourir les archives entière de manière satisfaisante, nous nous sommes fondé sur un premier travail effectué lors de notre recherche doctorale sur un corpus de presse sportive bilingue d'environ 400000 mots que nous avions construits et auquel un corpus parallèle construit à partir des archives du site Internet de l'UEFA de 70000 mots a été adjoint [Vanoudheusden : 2010, 96-101].

15 Après avoir recensé les principaux éléments <verbe + particule adverbiale> ressortis de nos recherches et qui ont pour signification la sortie, l'élimination, ou la mise à l'écart, nous avons utilisé les archives parallèles présentes sur les sites Internet, à savoir les traductions proposées à la publication les mêmes jours, afin d'y retrouver une traduction en français pour une étude contrastive.

(2) "Last summer in Germany, energised by the tens of thousands of supporters who crossed the border to encourage them, Switzerland came agonisingly close to reaching the quarter-finals, losing out on penalties against Ukraine." - UEFA, le $1^{\mathrm{er}}$ mars 2007.

(2') "L'été dernier en Allemagne, portée par des dizaines de milliers de supporteurs n'ayant pas hésité à traverser la frontière pour l'encourager, l'équipe de Suisse manquait d'un rien la qualification pour les quarts de finale de la Coupe du Monde de la FIFA (0-0, 0-3 t.a.b. face à l'Ukraine). » UEFA, le $1^{\text {er }}$ mars 2007.

Dans ces derniers exemples apparait une des premières conséquences de l'impossibilité de créer en français des compositions comme les verbes prépositionnels. En effet, un circonstant de manière doit compléter le verbe manquer afin de rendre le sens de lose out. 
Les emplois de verbes à particules spécifiques au sport se décomposent selon deux catégories : des emplois existants auxquels un sens dérivé à été donné; et des structures verbales dont seul un sens sportif est répertorié dans les dictionnaires, ou qui n'est pas répertorié, et même si cette dernière catégorie reste à la marge, elle montre à quel point la productivité de ce schéma offre aux journalistes sportifs une possibilité d'être particulièrement créatifs Les journalistes utilisent évidemment des formes qui appartiennent au langage courant, ou utilisent des formes verbales déjà existantes auxquelles ils ont prêté un sens approchant du sens originel. Ainsi, rule out signifie make it impossible for something to happen. Le phrasal verb n'est utilisé dans le DJS qu'avec un sujet inanimé représentant une blessure : injury en (3) et (4), le pronom relatif which ayant pour antécédent ligament damage en (5) ou sous forme passive ou adjectivée où l'agent est indiqué (6) ou absent (7).

(3) "Injury had ruled David Beckham out of the game, but he had the pitch to himself when he strode back on to the surface where he made his name during the 1990s." - UEFA, le 13 mars 2007.

(3') «Blessé, David Beckham ne pouvait prendre part à la rencontre, mais le stade lui était entièrement acquis lorsqu'il fit son apparition sur le pelouse qui marqua ses débuts dans les années 1990. » - UEFA, le 13 mars 2007.

(4) "SL Benfica's Rui Costa hopes injury will not rule him out of the UEFA Cup Round of 16 tie against Paris Saint-Germain FC as he looks to embellish his trophy collection." - UEFA, le 1er mars 2007.

(4'.) «Le milieu du SL Benfica Rui Costa espère qu'il ne manquera pas les huitièmes de finale de la Coupe UEFA, contre le Paris Saint-Germain FC, à cause d'une blessure. »- UEFA, le 1er mars 2007.

Le français, quant à lui, inverse la proposition faisant du sujet anglais injury un circonstant de cause (blessure) en position finale.

(5) "Manchester United manager Sir Alex Ferguson said Neville had suffered ligament damage and could be sidelined for up to three weeks, which would rule him out of England's qualifiers against Israel and Andorra."- UEFA, le 17 mars 2007.

(5') «Le manager de MU Alex Ferguson, a déclaré que Neville "souffre des ligaments". "Je pense qu'il sera absent pendant trois semaines." Le défenseur sera absent face à Andorre et à Israël avec l'Angleterre. » - UEFA, le 17 mars 2007.

(6) "Vincenzo Iaquinta, Fabio Grosso and Christian Zaccardo are all ruled out through injury, and Francesco Totti omitted by his own request." - UEFA, le 18 mars 2007.

(6') «Vincenzo Iaquinta, Fabio Grosso et Christian Zaccardo sont blessés et Francesco Totti a été laissé de côté sur sa propre demande. »- UEFA, le 18 mars 2007.

(7) "Martin Laursen, Thomas Helveg, Sören Larsen and Peter Lövenkrands have all been ruled out of the match on 24 March when the Danes take on Spain looking to close the gap on runaway Group F leaders Sweden." - UEFA, le 14 mars 2007.

(7’) « Martin Laursen, Thomas Helveg, Sören Larsen et Peter Lövenkrands ne prendront pas part à la rencontre du 24 mars qui verra le Danemark se mesurer à l'Espagne dans un Groupe F où la Suède a fait le trou. » - UEFA, le 14 mars 2007.

Les traductions marquent les différentes possibilités qui s'offrent pour l'expression des verbes prépositionnels en français. Tous les exemples français doivent traduire la notion portée par out. L'exemple (5') met en avant l'affixation avec ab-sent. En (3') et (7'), le 
français fait le choix de la négation du contraire de l'expression prendre part. En (4'), le sens de l'éviction est présent par le choix lexical du verbe manquer désignant sémantiquement une conséquence. Enfin, en (6'), la traduction française ne se focalise pas sur la conséquence (mise à l'écart des joueurs pour raison d'impossibilité physique) mais uniquement sur la cause de cette mise à l'écart : Vincenzo Iaquinta, Fabio Grosso et Christian Zaccardo sont blessés, induisant ainsi l'absence.

Nous avons également relevé l'occurrence de play out suivi d'un syntagme nominal signifiant un match nul. L'OED note que play out signifie bring to an end et donne une définition spécifique au cricket keep playing until the end of (the appointed time). Les usages en (8) et en (9) sont donc différents de ceux recensés par les lexicographes. Les deux traductions françaises se quitter/se séparer sur un score nul/dos à dos donnent une traduction qui dénote la fin d'un match et la séparation des équipes sous forme de verbes pronominaux avec un complément circonstanciel de manière (la locution adverbiale dos ̀̀ dos et le groupe prépositionnel sur un score de 1-1). Encore une fois, le français traduit l'élément sémantique le plus important, c'est-à-dire la particule adverbiale out, soit sémantiquement le mouvement, mais ne peut traduire le verbe support qui exprime le moyen, sous peine de lourdeur.

(8) "The United Kingdom rivals played out a goalless draw at Windsor Park in Belfast, a result due in no small part to the absence of attacking talent on both sides." - UEFA, le 6 mars 2007.

(8') «Les rivaux du Royaume-Uni se sont quittés dos à dos au Windsor Park de Belfast, le score traduit les carences offensives des deux équipes. »- UEFA, le 6 mars 2007.

(9) “RSC Anderlecht coach Frank Vercauteren described Sunday's meeting with leaders KRC Genk as a battle after Belgium's top two played out a gruelling 1-1 draw in Genk to keep the title race wide open." - UEFA, le 12 mars 2007.

(9') « L'entraîneur du RSC Anderlecht Frank Vercauteren a comparé le choc de dimanche face au leader du KRC Genk à une bataille, les deux meilleures équipes du championnat s'étant séparées sur un score de 1-1 à Gand. La course au titre est plus ouverte que jamais. »- UEFA, le 12 mars 2007.

De même, to sit out (10) et (11) est traduit en français par manquer un match. Pour les mêmes raisons que précédemment, le français ne peut traduire entièrement la structure qui signifie to remain seated, so as take no part in a game. Dans la traduction, on se focalise donc sur l'exclusion temporaire.

(10) “The English international, who will also sit out his country's UEFA EURO 2008' qualifying Group E games against Israel and Andorra at the end of this month, is expected to be on the sidelines for three weeks after having the operation to cure a persistent injury." - UEFA, le 14 mars 2007.

(10') «L'international anglais manquera également les rencontres de son pays contre Israël et Andorre dans le Groupe E des éliminatoires de l'UEFA EURO 2008' à la fin du mois. » - UEFA, le 14 mars 2007.

(11) "Miroslav Klose and Clemens Fritz are suspended for the 24 March match in Prague, and will also sit out the friendly against Denmark four days later, although Fritz is on standby for the game in Duisburg." - UEFA, le 16 mars 2007.

(11') « Miroslav Klose et Clemens Fritz sont suspendus pour la rencontre du 24 mars à Prague et manqueront le match amical contre le Danemark à Duisbourg, quatre jours plus tard. Toutefois, rien n'est encore sûr concernant Fritz pour ce match. » - UEFA, le 16 mars 2007. 
17 Le même problème se pose pour la traduction de clear off et de chalk off. Le premier, dans cet emploi spécifique, est une variante du même phrasal verb que l'on trouve par exemple dans They cleared off when they saw the police coming, façon informelle de dire qu'ils ont quitté un lieu très vite, et traduit en français par le verbe pronominal informel se tirer. Ici, le sens s'approche plutôt de clear up, signifiant ranger, rendre un endroit plus net, mais le journaliste utilise la particule adverbiale off pour signifier que le ballon a été dégagé, terme dont on peut encore une fois noter la construction affixale.

Quant à chalked off, il s'agit d'un emploi spécifique au sport pour signifier que le but n'a pas été validé par l'arbitre, étymologiquement qu'il a été effacé du tableau indiquant les scores. On retrouve une certaine logique à traduire dans les deux cas par un élément affixé, même si le deuxième terme est tellement lexicalisé que le préfixe n'est sûrement plus perçu comme tel.

(12) "Diego and Naldo then went close to making the tie safe, the latter with an attempt cleared off the line, before Celta substitute Jesus Perera saw the first of two 'goals' chalked off for offside." - UEFA, le 14 mars 2007.

(12') « Diego et Naldo étaient tout proches du but. Le second voyait sa frappe repoussée sur la ligne, avant que le premier «but» de Jesus Perera ne soit refusé pour une position de hors-jeu. » - UEFA, le 14 mars 2007.

Ces exemples laissent envisager une première catégorisation possible selon la lexicalisation des schémas de composition particulièrement anciens et sans aucun doute désormais plus ressentis comme étant des métaphores; des schémas de composition spécialisés et uniquement acceptés dans des domaines précis comme ici celui du sport ces derniers étant probablement relevés comme moins saillants par des habitués de ce type de discours que par un public non-spécialiste.

Parmi les occurrences rencontrées ${ }^{1}$, nous trouvons évidemment des verbes supports classiques tels que get, take ou encore send. Ceux-ci, dont le sémantisme se prête particulièrement à la composition, sont nombreux, et probablement peu reconnu comme étant les verbes supports de verbes métaphoriques par les anglophones. Par contre, il apparait certaines occurrences comme celle de l'exemple (12) qui laissent supposer que la création lexicale dans un domaine spécifique tel que celui étudié est moins figée, et donc qu'il doit y avoir une incidence particulière quant à la traduction - et c'est ce que nous verrons dans les prochains exemples. De plus, et sans aucun doute, la saillance métaphorique est plus importante et donc particulièrement plus ressentie tant par les lecteurs que par les traducteurs, dans les constructions métaphoriques dont le verbe support n'est pas un light verb habituellement utilisé dans la langue anglaise.

Tous les exemples présentés précédemment ont permis d'illustrer les disparités de système entre les deux langues et des implications que celles-ci peuvent avoir sur le passage d'une langue à une autre, et en particulier de l'anglais au français dans le cas des verbes prépositionnels.

\subsection{Composition et affixation}

Les exemples qui suivent sont des termes que nous pourrions considérer comme terminologiques, et qui du moins, sont le résultat d'une évolution sémantique due à la spécificité du domaine sportif. Il y a alors une adaptation au domaine mais pas pour autant de manière automatique. Si l'équivalent de knock out est souvent « éliminer », que 
l'on se trouve dans une situation spécifique au sport ou plus générale, l'automatisation de la traduction n'est pas possible pour des mémoires de traductions quand il s'agit par exemple du verbe bow suivi de la particule out. Celle-ci a comme définition générale « leave a job or stop doing an activity, usually after a long time » ce qui explique la traduction en (1) ou bien celle en (13) mais pas celle en (14).

(13) Spain scored five goals in total and finished second in the group behind Germany, its two wins and a draw from three matches good enough to knock reigning Olympic champions Australia out of the tournament and to qualify for the semi-final stage. - CIO, le 8 septembre 1992.

(13') L'Espagne marqua cinq buts au total et termina deuxième du groupe, juste derrière l'Allemagne, éliminant l'équipe australienne, championne olympique en titre et se qualifiant pour les demi-finales. - CIO, le 8 septembre 1992

(14) [...] while Fogelstrom top scored with five for Sweden, who bow out of the competition having lost all four of their games. - UEFA, le 3 août 2008.

(14') [...] alors que Fogelstrom a été la meilleure marqueuse suédoise avec cinq buts pour son équipe, qui est éliminée de la compétition après avoir perdu ses quatre matchs. - UEFA, le 3 août 2008.

Pour autant, dans les deux cas de traduction, on se retrouve avec les deux éléments classiques de la composition verbale dans les systèmes linguistiques en concurrence. La langue anglaise a comme nous l'avons déjà rappelé une tendance importante à la création de verbes à particules, donc à la composition, suivant ici un schéma verbe + préposition, dont bow out est prototypique. En français, la traduction par éliminer l'est tout autant, car elle met en avant la tendance des langues latines à la création lexicale par affixation.

La traduction française opte toujours dans nos exemples pour le verbe éliminer. Ceci marque une des différences classiques entre français et anglais dans la formation morphologique des verbes :

Comme les autres composé anglais par juxtaposition, les phrasal verbs correspondent fréquemment à des dérivés par affixation en français. [Chuquet \& Paillard $1989: 199]$

L'anglais, avec knock out fait le choix de la composition <verbe + particule adverbiale> alors que le français privilégie l'affixation étymologique d'origine latine ex-liminare sans pouvoir rendre le moyen (knock). Le même phénomène apparait dans les exemples (15)(15') avec la composition anglaise washed away traduite par submergé, <sub-merge>.

(15) "[...] washed away by a storm that answers to the name Jo-Wilfried." The Times, le 25 janvier 2008.

(15’) « Tsonga a submergé le triple vainqueur de Roland-Garros »- Le Monde, le 26 janvier 2008.

\subsection{Traduction directe}

Parmi les traductions de métaphores anglaises utilisées dans le domaine du sport, il convient désormais de se pencher sur les différentes opérations de traduction. Nous avons pu constater dans les exemples précédents que les traducteurs essaient, dans la mesure du possible, de rester le plus près possible du texte source, et ce, probablement à cause d'une contrainte de temps qui veut que les articles soient publiés en même temps. En amont, une autre contrainte temporelle s'ajoute à celle-ci, car les journalistes rédacteurs de ces articles subissent une même pression qui les oblige à écrire rapidement 
afin de publier le plus tôt possible après la fin d'un événement sportif. Ce phénomène s'est encore accentué de manière exponentielle avec l'information en continu et sa nécessité d'immédiateté dans un premier temps puis les choix éditoriaux des organes de presse d'une plus grande présence sur Internet et enfin la possibilité via les applications mobiles de recevoir l'information en tout temps et en tout lieu.

L'opération de traduction qui est donc la moins couteuse semble être celle qui ne nécessite aucun ou le moins possible de changements au niveau syntaxique. En somme, il est nécessaire de privilégier les procédés directs de traductions comme la traduction littérale.

Dans le cadre de cette analyse, la plus directe des traductions est donc celle qui pourrait être symbolisée comme suit : $\mathrm{Vb}+$ particule (angl.) $\Rightarrow \mathrm{Vb}$ (fr.)

(16) "Another mix-up enabled Massimo Ambrosini to pick out Klaas Jan Huntelaar, who drove just wide." - UEFA, le 16 février 2010.

$\left(16^{\prime}\right)$ «Un autre cafouillage au milieu de terrain permettait à Massimo Ambrosini de trouver Klaas Jan Huntelaar qui frappait à côté. » - UEFA, le 16 février 2010.

Dans les exemples précédents et suivants, pick out représente l'objectif du joueur quand il effectue une passe. En anglais, le journaliste se focalise donc sur la possibilité événementielle de faire ce choix (16). Dans nos exemples en anglais, la visée est donc à chaque fois présente via la particule to. En français, l'exemple (16') utilise le verbe trouver qui dans le domaine des sports collectifs de ballon signifie réussir à passer le ballon à untel.

En anglais, la traduction littérale find est également courante.

The verb to find is central [in our results], but to feed, to release, to strike, to pick out, to look for, to set up, to play on and to put in are also in connection with a player as direct object. [Krone 2005: 104]

(17) "The home side were struggling to pick out Óscar Cardozo until Di María finally combined with his colleague on 33 minutes, floating a ball over the defence that Cardozo volleyed into the stands." - UEFA, le 11 mars 2010. (17') «Benfica avait du mal à trouver Óscar Cardozo jusqu'à une belle combinaison avec Di María à la 33e minute. Mais la volée de Cardozo échouait dans les tribunes. » - UEFA, le 11 mars 2010.

La traduction en (18) porte le même sens, mais pick out est traduit par le verbe " centrer ", qui est spécifiquement une manière de faire une passe. La traduction française est plus spécifique avec la circonstancielle de manière à ras de terre. On arrive ici à une des limites de la traduction de ce genre de texte et de groupes verbaux spécifiques. Le texte source est le (18) mais le traducteur a probablement vu les images de l'action, ce qui lui a permis de compléter le texte. Dans l'exemple (18'), l'étoffement est probablement rendu obligatoire par le fait que pick out et lay the ball off to soient tous les deux des termes traduits en français par passer.

(18) "Paul Konchesky was sent clear down the left and he had time to pick out Bobby Zamora, who laid the ball off to the onrushing Damien Duff to strike low beyond Diego Benaglio in the Wolfsburg goal." - UEFA, le $1^{\mathrm{er}}$ avril 2010.

(18') « Konchesky s'infiltrait à gauche, centrait à ras de terre pour Zamora qui déviait en douceur pour Duff, dont la frappe à l'entrée de la surface ne laissait aucune chance au portier de Wolfsburg. » - UEFA, le $1^{\text {er }}$ avril 2010. 
(19), lay the ball off to est traduit par alerter. Il s'agit d'une utilisation spécifique au football qui signifie « frapper » le ballon dans le but de faire une passe dans la course d'un joueur de la même équipe. Le passage de l'anglais au français (19') nécessite l'utilisation d'un verbe transitif avec le nom propre Berbatov en complément d'objet direct alors que le verbe anglais avait deux compléments d'objet.

(19) "The game was up for the Portuguese side in the $76^{\text {th }}$ minute, however, when Malbranque laid the ball off to Berbatov and the No9's lovely flick let in the midfielder to complete the scoring." - UEFA, le 14 mars 2007.

(19') «L'affaire était toutefois entendue pour l'équipe lusitanienne à la 76e minute. Malbranque alertait Berbatov et le numéro 9, altruiste, permettait au milieu de terrain de clôturer le score. » - UEFA, le 14 mars 2007.

d'une passe. La passe en elle-même étant un geste anodin dans le football, le fait qu'elle mène à un but semble pousser le journaliste à «l'embellir ». C'est une tendance assez importante dans le journalisme sportif, mais elle est ici le résultat tant de ce que nous appelons «l'hyperbolisation du discours " [Vanoudheusden 2013], que de contraintes dues aux systèmes linguistiques. Il existe clairement dans le cadre de la mise en mot de l'événement "passe » des différences liées aux structures morpho-syntaxiques : l'anglais décrit le mouvement quand le français se focalise sur la communication établie entre les joueurs.

même, alors qu'on trouve très souvent une sorte de correspondance entre knock out et se faire/être éliminé(e) comme nous le verrons plus tard, l'exemple suivant relève d'une certaine recherche d'un terme plus sophistiqué pour parler d'une défaite (s'incliner) :

(20) Sánchez-Vicario was part of the Olympic tennis tournament in 1988, when tennis was included again in the Olympic programme. She was, however, knocked out in the first round. - CIO, http://www.olympic.org/ arantxa-sanchez-vicario

(20') Deux ans plus tard, en 1988, elle fait partie des 20 meilleures joueuses mondiales et participe au tournoi olympique de tennis qui fait alors sa réapparition au programme officiel des Jeux après 64 ans d'absence. Arantxa s'incline cependant dès le $1^{\mathrm{er}}$ tour. - CIO, http://www.olympic.org/fr/ arantxa-sanchez-vicario

\subsection{Verbe sous forme de participe}

Mais entre également en compte un critère qui jusqu'ici n'a pas été abordé qui est celui des conventions d'écritures. Ceci est particulièrement valables pour les titres d'articles dont nous verrons quelques exemples ici, sans pour autant qu'ils soient considérés comme prototypiques. Mais force est de constater que ces exemples mènent à une conclusion quant au procédé de traduction mis en place, qui n'est plus dans ces cas direct mais oblique, puisqu'ils forcent à un changement de catégorie lexicales.

Le cas rencontré le plus fréquemment est la traduction du verbe par un adjectif, souvent formé par le participe d'un verbe en français.

(21) Ba lifts Chelsea as Newcastle bow out. - UEFA, le 5 janvier 2013.

(21') Ba décisif, Newcastle éliminé. - UEFA, le 5 janvier 2013.

(22) Braga pair bow out with heads held high., - UEFA, le 19 mai 2011

(22') Braga battu mais tête haute. - UEFA, le 19 mai 2011 
Cette conversion implique donc des altérations de la phrase au niveau de la syntaxe, ce à quoi il faut ajouter dans ces deux cas le fait qu'il s'agisse de titre d'articles, ce qui a des conséquences sur la forme, à cause entre autre, de la recherche de concision alliée à la volonté d'incitation à la lecture. En (21') et (22'), ces contraintes mènent au choix de l'expression d'un contraste jouant sur la polarité, contraste autant présent dans les titres en anglais qu'en français. Le titre français en (21') ne donne pas tout à fait les mêmes informations que le titre anglais. Mais, il faut prendre en compte l'importance du contexte des articles sur le sport dans la presse écrite (classique ou Internet) qui veut que les journalistes s'adressent en priorité à un lectorat de spécialistes qui connaît potentiellement déjà l'information, et qui cherche du commentaire. Ainsi en (21'), le seul nom de Ba permet, pour les connaisseurs, d'identifier l'équipe pour laquelle il a été décisif. De même, en (22'), la traduction en français a eu pour conséquence une adaptation de l'information, avec un choix de ne plus se focaliser sur les deux joueurs protagonistes de l'article. Dans des termes de traduction, l'intérêt relève particulièrement du passage de bow out en un verbe dans son mode adjectival, « éliminé » et "battu ». Quant aux traductions suivantes, elles marquent l'autre possibilité offerte par la traduction en français de garder le verbe mais dans un mode non actif, dans une participiale.

(23) The player deserved all the praise he got for his enterprising showing too, striding forward commandingly with the ball, hitting snappy passes and always looking to pick out unmarked team-mates. - FIFA, le 14 décembre 2012.

(23') Il faut reconnaître que le joueur s'est bien acclimaté à ce poste, dynamisant la sortie de balle des Blues avec ses démarrages et ses passes rapides, et recherchant souvent ses coéquipiers en profondeur. - FIFA, le 14 décembre 2012.

(24) "The new coaching team proved their worth by knocking Madrid out of the UEFA Champions League first knockout round on away goals and restoring stability to the club's league results, although Bayern still trail Bundesliga leaders FC Schalke 04 by six points." - UEFA, le 15 mars 2007.

$\left(24^{\prime}\right)$ «Le nouveau tandem a déjà fait forte impression en éliminant Madrid lors du premier tour à élimination directe de l'UEFA Champions League (sur les buts en déplacement) et en remettant le club en selle dans le championnat allemand, même si le Bayern a toujours 6 points de retard sur les leaders du FC Schalke 04. » - UEFA, le 15 mars 2007.

\subsection{Verbe à la forme passive}

Dans l'exemple suivant et sa traduction, bow out est traduit par la forme se faire éliminer. La traduction de la forme verbale en V-ing est rendue par une construction syntaxique complexe avec la gérondive étant donné que introduisant une complétive. L'emploi dans l'exemple (25) est une dérivation du sens spécifique au sport puisque l'arrêt dans la compétition est une sanction rarement souhaitée par les membres des équipes.

(25) "With FC Dinamo 1948 Bucaresti bowing out against SL Benfica in the Round of 32 , the Uruguayan striker has now seen his main rival in the competition - fellow eight-goal striker Claudiu Niculescu - fall out of contention, and a goal in Espanyol's Round of 16 tie against Maccabi Haifa FC would now see 'El Rifle' claim top spot for himself." - UEFA, le 6 mars 2007.

(25') «Étant donné que le FC Dinamo 1948 Bucuresti s'est fait éliminer par le SL Benfica en seizièmes de finale, l'Uruguayen a vu son plus sérieux rival, le 
buteur Claudiu Niculescu, également auteur de huit réalisations depuis le début de l'épreuve, se faire écarter. Si Pandiani ajoute une unité à son total face au Maccabi Haifa FC, il prendra seul la tête du classement des buteurs. » - UEFA, le 6 mars 2007.

En (25), nous nous sommes également intéressé à la traduction de fall out of contention qui signifie ne plus avoir une chance de gagner. En français la traduction par la structure pronominale causative se faire écarter (de la compétition) insiste sur le résultat du procès (l'élimination) alors qu'en anglais il ne s'agit que du résultat du verbe fall. Ces propositions sont également de nature différente puisque l'anglais choisit un groupe prépositionnel circonstant alors que le français utilise une subordonnée complétive dans une participiale.

\subsection{Adaptation}

Les emplois suivants sont anodins quant à la sémantique des verbes en anglais mais montrent tout de même la difficulté du passage en français. Edge out signifie battre quelqu'un avec une petite marge. Dans l'exemple (26)-(26'), la forme passive du titre anglais permet d'élider l'agent, donc l'équipe qui a battu l'Energie Cottbus d'un but, afin de se concentrer sur l'équipe perdante, sujet de l'article. En français, le choix de ne pas traduire le titre anglais mais d'en créer un sur la base d'un jeu de mot humoristique a été privilégi :

(26) "Energie edged out" - UEFA, le 18 février 2007.

(26') « L'Energie en panne » - UEFA, le 18 février 2007.

Pour autant, nous considérons qu'il s'agit d'un exemple à la marge que l'on peut considérer comme une adaptation de point de vue, puisqu'un club battu est un club qui n'avance pas en termes de points dans un championnat.

(27) "It was the Cameroonian international striker who sparked Frank Rijkaard's team back into life at the Stade de France last May, cancelling out Sol Campbell's first-half header and starting the comeback which culminated in Juliano Belletti depriving Arsenal FC of the European Champion Clubs' Cup." - UEFA, le 6 mars 2007.

(27') «C'est l'international camerounais qui ravivait les espoirs des hommes de Frank Rijkaard au Stade de France en mai dernier. Il égalisait après l'ouverture du score de Sol Campbell en première mi-temps. » - UEFA, le 6 mars 2007.

L'emploi de cancel out est une adaptation du sens général au domaine du sport. Ce phrasal verb signifie annuler des forces. Dans le cadre sportif, et dans l'exemple (27), il signifie égaliser, comme traduit en (27') soit annuler l'avantage de l'équipe adverse. La traduction française, une fois encore, montre la prédilection de la langue française à utiliser l'affixation <égal + suffixe verbal -ise> alors que l'anglais choisit la composition <cancel + particule adverbiale out>.

La série d'exemples suivants sont tous traduits par la même locution verbale en français, quitter la zone de relégation (la place de lanterne rouge). Tous les exemples parlent d'un club de football qui, grâce à une victoire, gagne une ou des places dans le classement et n'est donc plus provisoirement relégable. L'exemple (31') donne une variante plus spécifique, puisqu'il ne s'agit plus de quitter la zone de relégation (en général les deux ou trois 
derniers au classement) mais la dernière place au classement, couramment appelée la lanterne rouge. Ce qui nous intéresse particulièrement dans cette série d'exemples c'est de voir que la langue française traduit toujours par le même verbe des formes verbales anglaises différentes. Ainsi, move out (28), jump out (29), climb ou (30) et climb off (31) portent tous le sens d'un déplacement vers l'extérieur de cette zone. En (29), (30) et (31), le choix lexical des verbes supports dénote un effort plus important de la part de l'équipe qui se traduit par la métaphore du bond en dehors de la zone (29) et de l'ascension vers le haut du classement (30) et (31).

(28) "The best result of the day was achieved by Reggina Calcio as they won 4-1 at Calcio Catania to move out of the bottom three, a point above FC Messina Peloro who lost 3-0 at home to Torino FC." - UEFA, le 10 mars 2007.

$\left(28^{\prime}\right)$ «La meilleure opération de la journée a été réussie par la Reggina Calcio qui a surclassé le Calcio Catania 4-1 sur son terrain et a ainsi quitté la zone de relégation avec un point d'avance sur sur le FC Messina Peloro battu 3-0 à domicile devant le Torino FC. » - UEFA, le 10 mars 2007.

(29) "VfL Bochum 1848 jumped out of the Bundesliga relegation zone after a 3-1 win at DSC Arminia Bielefeld while FC Energie Cottbus remain in trouble after a 1-0 defeat at 1. FC Nürnberg." - UEFA, le 18 février 2007.

$\left(29^{\prime}\right)$ «Le VfL Bochum 1848 a quitté la zone de relégation de la Bundesliga en s'imposant 3-1 sur la pelouse du DSC Arminia Bielefeld, alors que le FC Energie Cottbus reste en danger après sa défaite 1-0 chez le FC Nürnberg. » UEFA, le 18 février 2007.

(30) "Aachen climbed out of the relegation zone and into 13th place after a 2-1 comeback success at home to 1. FSV Mainz 05." - UEFA, le 4 mars 2007.

(30') « Aix-la-Chapelle a quitté la zone de relégation et grimpé à la treizième place grâce à son succès 2-1 à domicile devant le FSV Mainz 05. »- UEFA, le 4 mars 2007.

(31') 'Elsewhere, Wigan Athletic FC and Reading FC were both held to goalless draws by Fulham FC and Portsmouth FC respectively, while, in the evening's late kick-off, West Ham United FC climbed off the bottom after winning 2-1 against Blackburn Rovers FC." - UEFA, le 17 mars 2007.

(31') « Ailleurs, le Wigan Athletic FC et le Reading FC ont tous deux concédé des scores nuls et vierges, respectivement face au Fulham FC et au Portsmouth FC, alors que, dans le match de la soirée, le West Ham United FC a quitté la place de lanterne rouge en s'imposant 2-1 face au Blackburn Rovers FC. » - UEFA, le 17 mars 2007.

Toujours relatifs au classement, les exemples (32) et (33) dénotent l'idée que les deux clubs se sont sortis du bas du classement. C'est un sens spécifique au sport répertorié dans l'OED : to establish a decisive lead over an opponent or opposing team dont (32') donne une traduction littérale. La traduction proposée en (33') ne se concentre pas sur l'action décrite en anglais mais sur une conséquence : l'adaptation pour sur le fait que les deux clubs personnifiés sont à l'abri d'une relégation en division inférieure, et peuvent donc respirer :

(32) “Frédéric Kanouté's goal against RC Celta de Vigo saw him pull away from the pack at the top of the ESM Golden Shoe rankings though, after a hat-trick at the weekend, SC Heerenveen's Afonso Alves is hot on his heels." UEFA, le 19 mars 2007.

(32') « Le but inscrit par Frédéric Kanouté face au RC Celta de Vigo l'a vu prendre l'avantage sur ses adversaires en tête du classement du Soulier d'or ESM, talonné par le buteur du SC Heerenveen, Afonso Alves, auteur du coup du chapeau ce week-end. » - UEFA, le 19 mars 2007. 
(33) "Energie and Aachen pull away" - UEFA, le 4 mars 2007.

(33') «Cottbus et Aix respirent » - UEFA, le 4 mars 2007.

\subsection{Conversion}

(34) In the day's first encounter, Zakaria Labyad rescued a point for Morocco as they drew 2-2 with Honduras, despite having Zakarya Bergdich sent off in the opening Group D fixture at Glasgow's Hampden Park. - CIO, le 26 juillet 2012.

(34') Dans la première rencontre de la journée, Zakaria Labyad a donné le point qui a sauvé le Maroc après un ex aequo, 2 à 2 , contre le Honduras, et ce malgré l'expulsion de Zakarya Bergdich en ce match d'ouverture du groupe D au stade de Hampden Park à Glasgow. - CIO, le 26 juillet 2012.

(35) " [...] their task was made all the easier seven minutes after the restart when Larsson was sent off." - CIO, le 30 juillet 2012.

(35') «Leur tâche n'en fut que facilitée par l'exclusion de Larson, sept minutes après la reprise. »-CIO, le 30 juillet 2012.

Ces deux exemples montrent que dans certains cas, qui semblent ne pas être fréquents, et qui semblent se cantonner à la paire «sent off - exclusion », le choix d'une conversion par le traducteur se fait systématiquement. La conversion d'un verbe vers un nom n'est pour autant pas rare dans ce sens de traduction, bien au contraire. Mais comme dans tous les autres cas de traduction, il implique de nombreux changements syntaxiques dans la phrase en français.

Enfin, dans un contexte où la traduction doit être rapide, et où les sources de texte et d'informations sur l'événement traités peuvent être nombreuses, le choix de l'ellipse totale ou de « l'oubli » en (36'), n'est que très peu fréquent. Dans l'exemple suivant, nous pourrions parler d'ellipse de la moitié du titre «as Russia bow out ». Mais, comme nous l'avons déjà vu précédemment, le journaliste et le traducteur s'adressent à des lecteurs qui connaissent l'information et qui peuvent ainsi déjà savoir que malgré le très bon match du joueur russe Shikorov, son équipe n'a pu se qualifier pour la suite de la compétition. Dans ce contexte où journalistes et lecteurs partagent déjà ce savoir commun, nous pouvons considérés que «non récompensés » qui portent sur les efforts du joueur, portent également le sens de la deuxième partie du titre.

(36) Shirokov efforts in vain as Russia bow out. - UEFA, le 17 juin 2012.

(36') Les efforts de Shirokov non récompensés. - UEFA, le 17 juin 2012.

\section{Conclusion}

41 Les exemples présentés dans cet article nous ont permis d'une part de montrer que le français et l'anglais n'utilisaient pas dans les mêmes cas l'affixation et la composition, mais surtout que les règles inhérentes aux systèmes linguistiques forcent également à une recomposition syntaxique lors de la traduction puisque le français a toujours tendance à externaliser les informations par des subordonnées quand l'anglais les internalise, par la composition adjectivale par exemple.

Le schéma de création lexicale « verbe support + expression du mouvement pour signifier l'exclusion » semble laisser libre court à la créativité des journalistes sportifs puisqu'ils peuvent, à volonté, user de verbes suivants ce schéma, soit en utilisant un verbe 
prépositionnel existant, soit en donnant à ces verbes un sens spécifique, soit en créant un terme également spécifique au domaine étudié.

Entre en jeu également le fait que, dans un domaine spécifique comme celui du commentaire sportif, les journalistes obéissent à des conventions stylistiques en anglais comme en français, qui forcent l'usage de termes hyperbolisant, en particulier les métaphores de l'exclusion en anglais suivant le schéma verbe + particule adverbiale.

Ces métaphores montrent l'éventail d'ingéniosité ou d'évitement que les traducteurs peuvent déployer, allant de la traduction directe à l'oblique avec adaptation, voire l'oubli.

Ces quelques pages permettent d'insister sur l'importance de persévérer dans ce sens, en s'appuyant sur une étude statistique qui permettrait d'une part de relever des éléments spécifiques par rapport à un corpus de textes journalistiques généralistes; d'autre part de corroborer certaines impressions perçues lors de ce début d'analyse.

\section{BIBLIOGRAPHIE}

CHUQuet Hélène \& PAILLARD Michel, Approche linguistiques des problèmes de traduction anglais <-> français, édition révisée, Gap, Paris : Ophrys, 1989.

KRONE Maike, The Language of Football. A Constrastive Study of Syntactic and Semantic Specifics of Verb Usage in English and German Match Commentaries, Stuttgart : Ibidem-Verlag, 2005.

LAKOFF George \& JoHnson Mark, Metaphors we live by, Chicago University of Chicago Press, 1980.

MicHELs Anke, "Metaphern in franzözichen Füßballreportagen”. Accessible à l'URL suivante : http:// www.metaphorik.de/de/journal/02/metaphern-franzoesischen-fussballreportagen.html, 2002.

NoRDIN Henrik, "The use of conceptual metaphors by Swedish and German football commentators - A comparison", in LAVRIC Eva et al. (eds.), The Linguistics of Football, Tübingen : Gunter Narr Verlag Tübingen, 2008.

ORWELl George, “The Sporting Spirit.” Tribune, n 468 (14 décembre), 1945 : 10-11.

SCHMIDT Thomas, "The Kicktionary : Combining corpus linguistics and lexical semantics for a multilingual football dictionary", in LAVRIC Eva et al. (eds.), The Linguistics of Football, Tübingen : Gunter Narr Verlag Tübingen, 2008.

TALMY Leonard, "Path to realization: a typology of event conflation", Proceedings of the Berkeley Linguistics Society 17, 1991: 480-591.

TOURNIER Jean, Précis de lexicologie anglaise, Paris : Ellipses, 2004.

VANOUDHEUSDEN Romain, Stéréotypes et variation sémantique dans un corpus de presse en anglais et en français, Thèse de doctorat, Université de Poitiers, 2010.

VANOUDHEUSDEN Romain, «Comme les journalistes sportifs nous parlent : étude d'un lexique « hyper-appréciatif », in ABECASSIS Mickael \& LEDEGEN Gudrun, Ecarts et apports des médias francophones, Oxford, Bern, Berlin, Bruxelles, Frankfurt am Main, New York, Wien : Peter Lang, $2013: 227-244$ 


\section{NOTES}

1. Dont voici la liste : bow out; cut out; kick out ; miss out; pick out; rule out; sit out; lose out ; play out ; bring off; lay off; climb off; send off; shake off; shrug off; take off; clear off; chalk off; get away; wash away; pull away.

\section{RÉSUMÉS}

$\mathrm{Au}$ vu de leur fréquence d'apparition et de leur emploi, les métaphores peuvent être considérées comme un des éléments principaux de la rhétorique des journalistes sportifs. Dans ce type de discours particulièrement structuré, les métaphores sont aussi un moyen de création lexicale particulièrement productif, qui représente un défi de traduction. Puisque le sport est une affaire de compétition, il est aussi, pour le perdant, une histoire d'élimination; pour cet article nous nous focaliserons sur une des constructions métaphoriques les plus courantes, le schéma particulièrement productif qui relève de la métaphore du mouvement traduisant une mise à l'écart, une exclusion ou une élimination, Verbe + out/off/away. À partir d'exemples tirés d'un corpus parallèle de brèves concernant des événements sportifs tirés de sites Internet des instances européennes et internationales, nous étudierons des exemples de constructions verbales anglaises suivant ce schéma et leurs traductions en français afin de montrer l'importante productivité de ce schéma d'unité lexicale en anglais ainsi que les difficultés éprouvées et les moyens mis en œuvre par les traducteurs lorsqu'il est question de rendre leur sens en français.

Metaphors are a productive means of lexical creativity. That is especially true in sports news commentaries, which is a particular textual genre in which many stylistic conventions are imposed upon sports journalists. This paper is a study of the construction "Verb + out/off/away" as a metaphor of elimination in English in such a context and its translation in French. It aims to show that the specific use of such metaphors in sports news articles published in English newspapers is a challenge for translators. Thus, the author is offering a range of different devices used to translate metaphors collected on the Internet (e.g. FIFA, UEFA and OIC websites). We intend to show how productive and creative this scheme is; what translators have to deal with and how they manage it.

\section{INDEX}

Mots-clés : métaphore, unités lexicales, langue à cadrage satellitaire, langue à cadrage verbal, traduction, commentaires de presse sportive

Keywords : metaphor, lexical units, satellite-framed language, verb-framed language, translation, sports news commentaries 


\section{AUTEUR}

\section{ROMAIN VANHOUDHEUSDEN}

Docteur de l'Université de Poitiers, France

romain.vanoudheusden@gmail.com 